\title{
La metodología Montessori en la educación física online: una experiencia en la educación inicial II
}

Montessori methodology in online physical education: an experience in initial education II

1 Ángel Aníbal Sailema Torres https://orcid.org/0000-0002-1670-004X Universidad Técnica de Ambato, Ambato Ecuador, aa.sailema@uta.edu.ec

2 Esmeralda Giovanna Zapata Mocha $\quad$ (iD) https://orcid.org/0000-0002-7439-452X Universidad Técnica de Ambato, Ambato Ecuador eg.zapata@uta.edu.ec

3 Silvia Beatriz Acosta Bones Universidad Técnica de Ambato, Ambato Ecuador silviabacostab@uta.edu.ec

4 Betty Paulina Tobar Lozada Universidad Técnica de Ambato, Ambato Ecuador betipautobar@hotmail.com

Sailema Torres, Ángel A., Zapata Mocha, E. G., Acosta Bones, S. B., \& Tobar Lozada, B. P. (2023). La metodología Montessori en la educación física online: una experiencia en la educación inicial II. ConcienciaDigital, 6(1.4), 1064-1078. https://doi.org/10.33262/concienciadigital.v6i1.4.2050

CONCIENCIA DIGITAL, es una Revista Multidisciplinar, Trimestral, que se publicará en soporte electrónico tiene como misión contribuir a la formación de profesionales competentes con visión humanística y crítica que sean capaces de exponer sus resultados investigativos y científicos en la misma medida que se promueva mediante su intervención cambios positivos en la sociedad. https://concienciadigital.org

La revista es editada por la Editorial Ciencia Digital (Editorial de prestigio registrada en la Cámara Ecuatoriana de Libro con No de Afiliación 663) www.celibro.org.ec 


\section{Palabras} claves: metodología Montessori, educación

física, modalidad online, educación inicial

Keywords:

Montessori methodology, physical education, online modality, initial
Resumen

Introducción. La aplicación de metodologías y estilos de enseñanza acorde con la edad e intereses de los escolares ofrece la oportunidad de incentivar su progreso cognitivo, motriz, del lenguaje y socioafectivo. En los menores de Educación Inicial potenciar su desarrollo desde el contexto de la Educación Física en modalidad online sigue siendo un gran desafío. Objetivo. Reflexionar sobre la importancia de la aplicabilidad de la Metodología Montessori en la clase de Educación Física online en el nivel inicial. Metodología. Se utilizó una metodología descriptiva, no experimental, con el empleo de métodos teóricos y empíricos. La población estuvo conformada por la totalidad del universo existente, integrado por estudiantes de 3 a 5 años, padres de familia y docentes de Educación General Básica de la Unidad Educativa Guayaquil. Resultados.1 Se sistematiza la importancia de la aplicabilidad de la Metodología Montessori en el nivel inicial para el desarrollo de las clases de Educación Física en modalidad online. 2.Quedó evidenciado que existen dificultades para el desarrollo de las clases de Educación Física por medio de la modalidad online, entre otros aspectos porque no todos los docentes dominan la metodología y formas adecuadas de enseñanza en esta modalidad y por otra parte porque los estudiantes que integran este nivel manifiestan desmotivación, temor ante la realización de actividades en modalidad online, así como dificultades de conectividad y acceso a la tecnología por lo que requieren acompañamiento familiar. 3. Es necesario capacitar a los docentes y padres de familia sobre el uso de la metodología Montessori. Conclusiones. La Metodología Montessori es fundamental para el desarrollo de las clases de Educación Física en modalidad online, permite disminuir las brechas existentes entre el nivel de competencias de los docentes y padres de familia y al mismo tiempo estimula la participación de los estudiantes en las clases.

\section{Abstract}

Introduction. The application of teaching methodologies and styles according to the age and interests of the schoolchildren offers the opportunity to encourage their cognitive, motor, language, and socio-affective progress. In initial education minors, promoting their development from the context of Physical Education in online 
education

\section{Introducción}

La aplicación de metodologías y estilos de enseñanza acorde con la edad e intereses de los escolares ofrece la oportunidad de incentivar su progreso cognitivo, motriz, del lenguaje y socioafectivo. En el caso de los menores que se integran a la Educación Inicial lo anterior reviste gran importancia; precisamente por las bondades que ofrece para su desarrollo integral la aplicación de métodos de enseñanza que se correspondan con sus necesidades e intereses.

Considerando las ideas planteadas, se destaca la utilidad de la aplicación de la Metodología Montessori para la estimulación temprana y el desarrollo de habilidades en los estudiantes del nivel inicial. Se coincide con Tobar (2021) al señalar que: "La metodología Montessori favorece la independencia, la libertad con límites y respeto por

mode continues to be a great challenge. Target. Reflect on the importance of the applicability of the Montessori Methodology in the online Physical Education class at the initial level. Methodology. A descriptive, non-experimental methodology was used, with the use of theoretical and empirical methods. The population was made up of the entire existing universe, made up of students from 3 to 5 years old, parents and teachers of Basic General Education of the Guayaquil Educational Unit. Results.1 The importance of the applicability of the Montessori Methodology is systematized at the initial level for the development of Physical Education classes in online mode. 2. It became evident that there are difficulties for the development of Physical Education classes through the online modality, among other aspects because not all teachers master the methodology and adequate forms of teaching in this modality and, on the other hand, because the students who integrating this level manifest demotivation, fear of carrying out activities in online mode, as well as difficulties with connectivity and access to technology for which they require family support. 3 . It is necessary to train teachers and parents on the use of the Montessori methodology. Conclusions. The Montessori Methodology is essential for the development of Physical Education classes in online mode, it allows reducing the existing gaps between the level of competencies of teachers and parents and at the same time stimulates the participation of students in the classes. 
el desarrollo físico y social del niño", enfatiza en su utilidad e importancia para el proceso de enseñanza-aprendizaje en modalidad online.

Según Bayod (2017) y Tobar (2021), la creación de un Aula de Montessori, además de ser necesario para el trabajo con escolares de estas edades, permite tener un espacio amplio, bonito, con buena iluminación y ventilación, de colores neutros que garantizan un ambiente agradable para el desarrollo del proceso de enseñanza-aprendizaje. Se trata de diseñar un espacio donde los niños puedan trabajar y aprender en situaciones similares a su casa, es decir un espacio que propicie el desarrollo de su imaginación, así como la mejora de su autonomía, el poder de decisión y elección acorde con sus gustos y preferencias.

En correspondencia con lo señalado la literatura describe los múltiples beneficios que tiene trabajar en la Educación Inicial siguiendo la Metodología Montessori, sin embargo, no siempre se logra motivar a todos los docentes para que apliquen la misma en el proceso de enseñanza-aprendizaje. En tal sentido se requiere profundizar sobre todo en el contexto de la Educación Física online.

Como se conoce con la llegada del COVID 19, fue necesario restructurar los procederes didácticos metodológicos que componen el proceso docente educativo y el de enseñanza aprendizaje en las diferentes asignaturas. En el caso de la Educación Física se ha tenido, al igual que en resto de las materias que integran el plan de estudio que migrar de la modalidad presencial a la online, con las consiguientes consecuencias que esto ha traído en una asignatura que de por sí, es eminentemente práctica, cabe preguntarnos. ¿Cómo incide la Metodología Montessori en la clase de Educación Física online en el nivel inicial? De ahí que la presente investigación centra su objetivo en: Reflexionar sobre la importancia de la aplicabilidad de la Metodología Montessori en la clase de Educación Física online en el nivel inicial.

\section{Un acercamiento a la Educación Física en modalidad online}

En la actualidad uno de los rasgos más evidentes de la Sociedad de la Información, el Aprendizaje Permanente y el Conocimiento es la omnipresencia de las TIC y la irreversibilidad de su penetración en todos los ámbitos de la vida. Los educadores físicos no podemos ignorar esa realidad ni abandonarnos de forma acrítica a su uso irracional e indiscriminado (Barahona, 2012; Tobar, 2021).

Se coincide con Sánchez et al. (2020), al señalar que el proceso de enseñanza en la Educación Física debe caracterizarse por la aplicación de varios estilos de enseñanza, los cuales deben ser dominados y seleccionados adecuadamente por parte del docente. En este sentido somos del criterio que, al tratarse de la práctica de actividades físicas con carácter formativo, el proceso de enseñanza-aprendizaje debe ser altamente significativo 
para los estudiantes que participan de la clase. Es por esta razón que la selección y aplicación de las metodologías y estilos de enseñanza requieren de un análisis previo que permita proyectar cómo se debe lograr el cumplimiento de los objetivos teóricos-prácticos a alcanzar durante el proceso de enseñanza-aprendizaje.

Para dar respuesta a lo anterior el docente que imparte la Educación Física, requiere poseer competencias genéricas y específicas que les permitan cumplir con la proyección realizada durante el proceso de planificación y desarrollo de la clase. Además, debe propiciar que en sus clases se dé cumplimiento al objetivo del Ministerio de Educación (2016), en el cual se destaca la necesidad de que los estudiantes alcancen el máximo desarrollo de sus capacidades, motivando la práctica sistemática de actividades físicas, que tributen a la formación integral de todos los estudiantes.

Como es lógico pensar con la llegada del COVID 19, lo planteado se ha convertido en un gran desafío; precisamente por la necesidad de que el profesor además de tener que aplicar nuevas y variadas metodologías y estilos de enseñanza, debe también motivar la participación de sus estudiantes, independientemente al nivel al cual asistan y a su vez garantizar que el proceso de enseñanza-aprendizaje sea significativo para los estudiantes que lo reciben. Al tener que impartir clases mediante la modalidad online los docentes se han visto en la necesidad de repensar cómo hacer este proceso atrayente para los estudiantes y cómo resolver las brechas presentes, dadas por la propia virtualidad en una asignatura que es eminentemente práctica.

Ello ha provocado que las clases han pasado de la presencialidad a la virtualidad, convirtiendo los grandes espacios, en espacios reducidos dentro del hogar para la realización de actividades físicas, deportivas y recreativas. Lo explicado ha traído serias consecuencias en cuanto al logro de objetivos, nivel de motivación y participación de los estudiantes, aplicación de nuevas formas y sistemas de evaluación de las destrezas y habilidades, así como en la propia calidad del proceso de enseñanza-aprendizaje de la Educación Física.

Según Prieto ( 2020) y Rioja (2020), si bien es cierto que con la pandemia se ha requerido buscar nuevas alternativas para continuar desarrollando las clases de Educación Física, coincidentemente con esto exponen las ventajas de la aplicabilidad de la tecnología y con ella de las redes sociales en el mantenimiento de estilos de vida saludables, entre otros aspectos por el acceso en línea a los diferentes programas que están disponibles en las diferentes plataformas dirigidos a orientar sobre la práctica de actividades físicas en condiciones de virtualidad.

En este orden de ideas Fuentes (2020) y Tobar (2021), demuestran en sus investigaciones que ante la pandemia se ha originado la necesidad en Educación Física como en otras asignaturas de cambiar la propuesta de enseñanza presencial a una estrategia de 
Educación Física online. Esto ha provocado la utilización y definición de un nuevo concepto basado en las tecnologías del aprendizaje y el conocimiento, el cual lo define como: "más innovador y actual", relacionándolo con todo lo concerniente al aprendizaje con el empleo de la tecnología. Al respecto Tobar (2021) señala que: "la introducción del campo virtual en el área de Educación Física es muy reciente y su incorporación es un reto para los docentes al suponer una innovación pedagógica que puede mejorar los procesos de adherencia a la actividad física"

Los estudios realizados por Posso et al. (2020), indican que en el contexto de la Educación Física en Ecuador acorde a los lineamientos curriculares se busca fomentar la autonomía en la actividad física, destacando la importancia de incorporar la virtualidad en las clases, desde enfoques inclusivos, lúdicos y de corporeidad. En consonancia con esto enfatizan en la urgencia de educar a los estudiantes en la práctica de actividades físicas desde la virtualidad, incluyendo todo lo referente con la labor educativa que debe darse dentro de la propia clase para fomentar en los estudiantes hábitos correctos de disciplina, cuidado y protección de los instrumentos de trabajo, buenas relaciones interpersonales entre otros temas.

Se coincide con Gómez et al. (2019), al señalar que independientemente a todos los desafíos presentados la Educación Física, se presenta como un área flexible y amoldable a todo lo relacionado con la virtualidad. Exponen los autores que existen variedades de recursos disponibles que pueden ser considerados y adaptados dentro del contexto de la Educación Física online. Siguiendo esta línea es preciso significar la idea de que estamos ante una situación que requiere atención y proyecciones, por tanto, es preciso saber hacer, pero hacer con criterio. Necesitamos una Educación Física que responda a los desafíos de los nuevos tiempos y establecer pautas que nos ayuden a integrarlas como un valor añadido capaz de enriquecer los procesos de enseñanza-aprendizaje.

Lo expresado implica que en la Educación Física online se debe determinar cuáles son los contenidos a enseñar tanto desde el punto de vista teórico como práctico y a su vez seleccionar adecuadamente las metodologías y estilos de enseñanza que permitan promover aprendizaje significativos y sustentables para la vida de los estudiantes.

Hay que modificar el hacer presencial por el hacer virtual, sin dejar en esto brechas que impidan evaluar el desempeño individual tanto de la adquisición de conocimientos teóricos como prácticos.

Educación física online en la educación inicial, desde la metodología Montessori

La educación Inicial comprende una etapa de grandes aprendizajes; es precisamente aquí donde se sientan las bases para el desarrollo integral; es por este motivo que resulta fundamental hacerla atrayente y significativa en toda su dimensión. 
La aplicación de metodología Montessori, representa una alternativa altamente beneficiosa en esta etapa del aprendizaje; las bondades que la componen hacen que se aplicable en cualquier asignatura.

En esta dirección los estudios realizados por Sailema et al. (2021), invitan a reflexionar sobre la factibilidad de esta metodología en los procesos de atención y estimulación multisensorial de niños con o sin necesidades educativas especiales, en condiciones de distanciamiento social

En el contexto de la Educación Física en el nivel inicial estudios precedentes realizados por Navacarrada (2010), Blández (2005), Estefó (2016) y Tobar (2021), reafirman los beneficios y utilidad de su aplicación.

En este sentido resultan demostrativos e interesantes los aportes relacionados con las ventajas de la metodología Montessori en torno a la creación de espacios de aprendizajes que resulten atrayentes y motivantes para los estudiantes. De igual manera se destaca su importancia en el proceso de estructura y diseño de las clases, en todo lo concerniente a la expresión de los movimientos, el desarrollo de aprendizajes mediante el juego, el diseño de actividades creativas y participativas y la creación de espacios didácticos que promuevan la independencia, la creatividad, la expresión y desempeño motriz y cognitivo de los niños

Fundamental resulta también las posibilidades que ofrece esta metodología al favorecer el desarrollo de las habilidades perceptivas del propio cuerpo, del entorno y habilidades y destrezas básicas.

Según criterios de los autores de esta investigación se puede plantear que estamos frente a una metodología que dada sus propias características y perspectiva debería ser considerada por todos los docentes, incluyendo los que imparten Educación Física en modalidad online. Los actuales programas de Educación Física en cualquier nivel de enseñanza requieren una clase que promueva el aprendizaje significativo desde las primeras edades, es por ello que la utilización de esta metodología requiere de preparación por parte de los docentes y del medio familiar máxime si la misma se empelará en condiciones de virtualidad.

\section{Metodología}

Se utilizó un enfoque mixto, con el empleo de una metodología de tipo descriptiva, de campo y documental, apoyados en métodos teóricos y empíricos. Es importante destacar que dada las condiciones de Pandemia la indagación y toma de datos se realizó mediante plataformas educativas como Teams, Zoom, herramientas office 365 y software libre, las cuales favorecieron el proceso de indagación y el trabajo con la muestra informante a través de la aplicación mediante Google Forms de la técnica de encuesta y de un 
cuestionario con preguntas cerradas para determinar aspectos importantes relacionados con la metodología Montessori en las clases de Educación Física.

La población estuvo conformada por la totalidad del universo existente, integrado por estudiantes de 3 a 5 años, 235 padres de familia y 11 docentes de Educación Inicial, 10 docentes de Educación Física, todos pertenecientes a la Unidad Educativa Guayaquil, ubicada Zona: 3 de la ciudad de Ambato.

\section{Resultados y Discusión}

A continuación, se exponen ejemplos de los resultados obtenidos de la aplicación de la Encuesta a Padres de Familia.

\section{Tabla 1}

Conocimiento sobre la ayuda que ofrece la metodología Montessori al proceso de aprendizaje teórico y práctico de la educación física

\begin{tabular}{|c|c|c|c|c|c|}
\hline \multicolumn{6}{|c|}{$\begin{array}{l}\text { 1. ¿Conoce usted si la Metodología Montessori ayuda en los procesos de aprendizaje } \\
\text { teórico y prácticos de la educación física? } *\end{array}$} \\
\hline & & Frecuencia & Porcentaje & $\begin{array}{l}\text { Porcentaje } \\
\text { válido }\end{array}$ & $\begin{array}{l}\text { Porcentaje } \\
\text { acumulado }\end{array}$ \\
\hline \multirow[t]{4}{*}{ Válido } & No & 45 & 19,1 & 19,1 & 19,1 \\
\hline & Poco & 86 & 36,6 & 36,6 & 55,7 \\
\hline & $\mathrm{Si}$ & 104 & 44,3 & 44,3 & 100,0 \\
\hline & Total & 235 & 100,0 & 100,0 & \\
\hline
\end{tabular}

Fuente: Tobar (2021)

En la presente pregunta, con la participación del $100 \%$ de encuestados, se puede ver que el 44,26\% manifestaron que, si conocen de la Metodología Montessori y de la ayuda en los procesos de aprendizaje teórico y prácticos de la Educación Física, mientras que el $36.60 \%$ poco conoce de la Metodología Montessori y de la ayuda en los procesos de aprendizaje teórico y prácticos de la educación física, y el 19,15\% dijeron de no conocen de la Metodología Montessori y de la ayuda en los procesos de aprendizaje teórico y prácticos de la educación física.

Esto indica que la mayor cantidad de padres de familia si conocen de la Metodología Montessori y de la ayuda en los procesos de aprendizaje teórico y prácticos de la educación física. 
Otra de las interrogantes realizadas fue la siguiente:

Tabla 2

Contribución de la metodología Montessori a las clases de educación física online

2. ¿Cree usted que la Metodología Montessori contribuirá a las clases de Educación Física online? *

\begin{tabular}{|c|c|c|c|c|c|}
\hline \multirow[b]{2}{*}{ Válido } & & Frecuencia & Porcentaje & $\begin{array}{c}\text { Porcentaje } \\
\text { válido }\end{array}$ & $\begin{array}{l}\text { Porcentaje } \\
\text { acumulado }\end{array}$ \\
\hline & A veces & 79 & 33,6 & 33,6 & 33,6 \\
\hline & No & 43 & 18,3 & 18,3 & 51,9 \\
\hline & $\mathrm{Si}$ & 113 & 48,1 & 48,1 & 100,0 \\
\hline & Total & 235 & 100,0 & 100,0 & \\
\hline
\end{tabular}

Fuente: Tobar (2021)

En la presente pregunta, con la participación del 100\% de encuestados, se puede ver que el 48,09\% manifestaron que, la Metodología Montessori si contribuye a las clases de Educación Física online, mientras que el 18.30\% dijeron que la Metodología Montessori no contribuirá a las clases de Educación Física online, y el 33,62\% dijeron de a veces la Metodología Montessori contribuirá a las clases de Educación Física online.

Esto indica que la mayor cantidad de padres de familia dicen que Metodología Montessori si contribuirá a las clases de Educación Física online.

La tercera pregunta realizada a los padres de familia buscó conocer si:

\section{Tabla 3}

Aplicación de actividades lúdicas y nivel que esto ayuda al desarrollo de los sentidos 3. ¿En la Metodología Montessori aplicamos actividades lúdicas-juegos en qué nivel considera usted que esto ayuda al desarrollo de los sentidos? *

\begin{tabular}{|c|c|c|c|c|c|}
\hline & & Frecuencia & Porcentaje & $\begin{array}{l}\text { Porcentaje } \\
\text { válido }\end{array}$ & $\begin{array}{l}\text { Porcentaje } \\
\text { acumulado }\end{array}$ \\
\hline \multirow[t]{4}{*}{ Válido } & Bajo & 18 & 7,7 & 7,7 & 7,7 \\
\hline & Medio & 107 & 45,5 & 45,5 & 53,2 \\
\hline & Alto & 110 & 46,8 & 46,8 & 100,0 \\
\hline & Total & 235 & 100,0 & 100,0 & \\
\hline
\end{tabular}

Fuente: Tobar (2021) 
En la presente pregunta, con la participación del 100\% de encuestados, se puede observar que el 46,8\% manifestaron que, es muy alto el desarrollo de los sentidos al aplicar actividades lúdicas en la metodología de Montessori, mientras que el $45.5 \%$ dijeron que es un nivel medio el desarrollo de los sentidos al aplicar actividades lúdicas en la Metodología de Montessori, y el 7.7\% dijeron de es muy bajo el desarrollo de los sentidos al aplicar actividades lúdicas en la Metodología de Montessori . Esto indica que los padres de familia manifestaron que es muy alto el desarrollo de los sentidos al aplicar actividades lúdicas en la Metodología de Montessori. Otra de las preguntas de interés se relacionó con los criterios respecto al aula Montessori, al respecto se consideró por parte de los encuestados lo siguiente:

\section{Tabla 4}

\section{El aula de Montessori}

4. ¿El Aula de Montessori es un espacio muy amplio, sencillo, bello y luminoso, con colores neutros, paredes con; posters y dibujos ya que se evita la sobre estimulación y la distracción, esto parece más una casa que un aula tradicional, ¿María Montessori "La casa de los niños” esto ayuda al desarrollo de la educación física?

\begin{tabular}{|c|c|c|c|c|c|}
\hline \multirow{4}{*}{ Válido } & & Frecuencia & Porcentaje & $\begin{array}{c}\text { Porcentaje } \\
\text { válido }\end{array}$ & $\begin{array}{l}\text { Porcentaje } \\
\text { acumulado }\end{array}$ \\
\hline & Poco & 7 & 33,3 & 33,3 & 33,3 \\
\hline & $\mathrm{Si}$ & 14 & 66,7 & 66,7 & 100,0 \\
\hline & Total & 21 & 100,0 & 100,0 & \\
\hline
\end{tabular}

Fuente: Tobar (2021)

En la presente pregunta, con la participación del 100\% de encuestados, se puede observar que el 66,7\% manifestaron que, el Aula de Montessori si es un espacio muy amplio, sencillo, bello y luminoso, con colores neutros, paredes con; posters y dibujos ya que se evita la sobre estimulación y la distracción, esto parece más una casa que una aula tradicional, María Montessori la denominó "La casa de los niños" esto ayuda al desarrollo de la educación física, mientras que el 33,3\% dijeron que el Aula de Montessori poco es un espacio muy amplio, sencillo, bello y luminoso, con colores neutros, paredes con; posters y dibujos ya que se evita la sobre estimulación y la distracción, esto parece más una casa que una aula tradicional, María Montessori la denominó "La casa de los niños" esto ayuda al desarrollo de la educación física, y el $0.0 \%$ dijeron que el Aula de Montessori a veces es un espacio muy amplio, sencillo, bello y luminoso, con colores neutros, paredes con; posters y dibujos ya que se evita la sobre estimulación y la distracción, esto parece más una casa que una aula tradicional, María Montessori la denominó "La casa de los niños" esto ayuda al desarrollo de la educación física, esto indica que el Aula de Montessori si es un espacio muy amplio, 
sencillo, bello y luminoso, con colores neutros, paredes con; posters y dibujos ya que se evita la sobre estimulación y la distracción, esto parece más una casa que un aula tradicional, María Montessori la denominó “La casa de los niños" esto ayuda al desarrollo de la educación física. En el caso de los resultados de la aplicación de las encuestas a docentes se pudo precisar que se reconoce por parte de algunos docentes que el método Montessori ayuda de una manera muy relevante al desarrollo físico y psíquico de los niños, con la aplicación de diferentes actividades creadoras, con las cuales se propone su integración para el desarrollo del aprendizaje basado en lo no tradicional, sin embargo, no todos los docentes reconocen su aplicación e importancia, planteando que en ocasiones no es la adecuada para la edad o la manera que se está impartiendo la clase.

Al realizar la revisión de los datos obtenidos en las encuestas se determina la necesidad de contar con una capacitación integral sobre el método Montessori a los padres de familia y docentes para aprovechar su aporte en el aprendizaje y desarrollo de los niños tanto internamente como externamente. La falta de conocimiento del método, así como el poco compromiso de los padres en el desarrollo de los niños por diferentes situaciones, nos permite tener como necesidad la aplicación de instrumentos, como de programas y métodos para mejorar el desarrollo de los niños. Los docentes deberán contar con un plan que contribuya a su crecimiento tanto profesional como personal y así mejorar su desempeño dentro de todos los roles que estos desempeñen.

\section{Tabla 5}

\section{Comprobación de la Hipótesis}

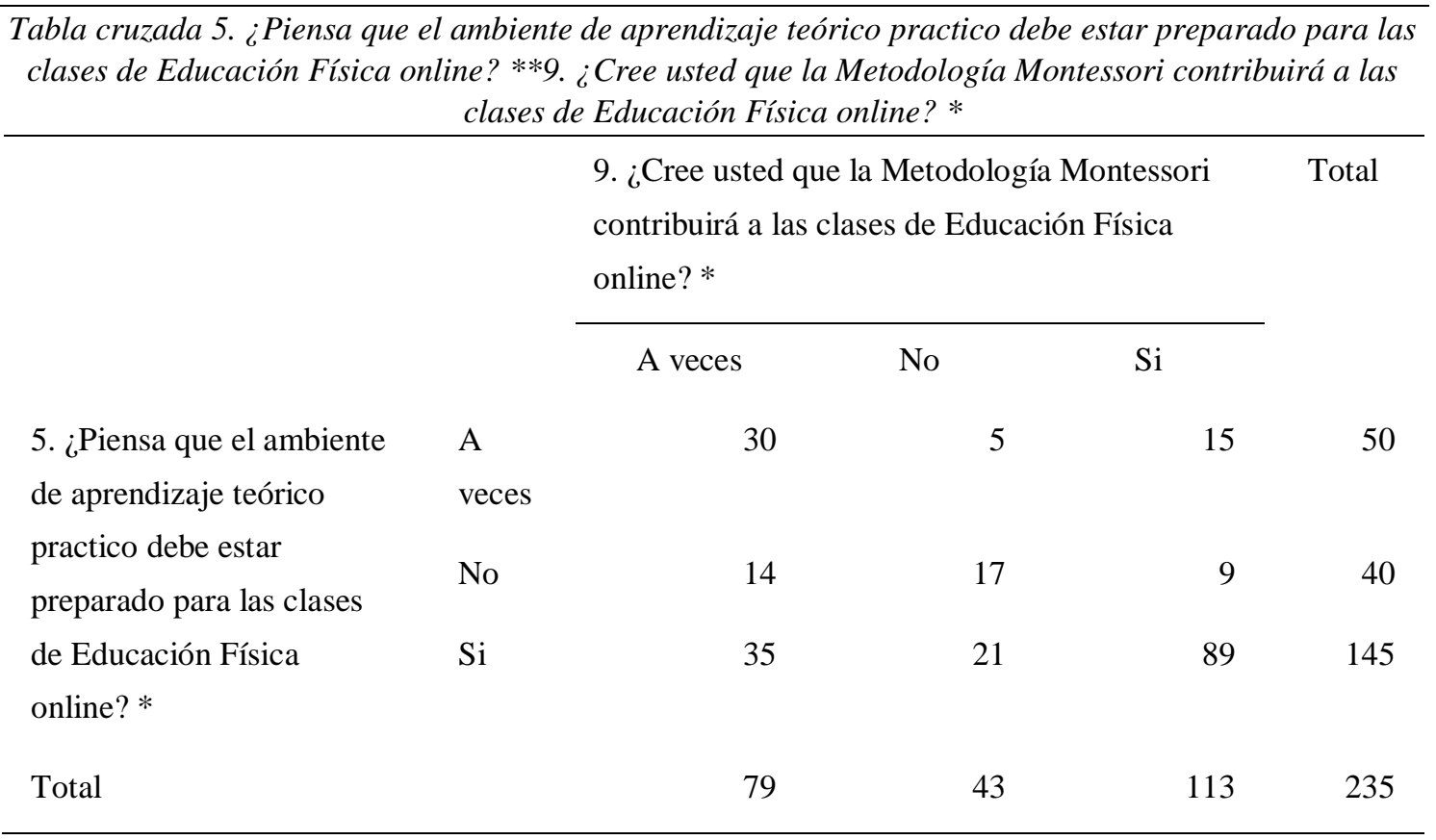

Fuente: Tobar (2021) 


\section{Figura 1}

Verificación de la Hipótesis

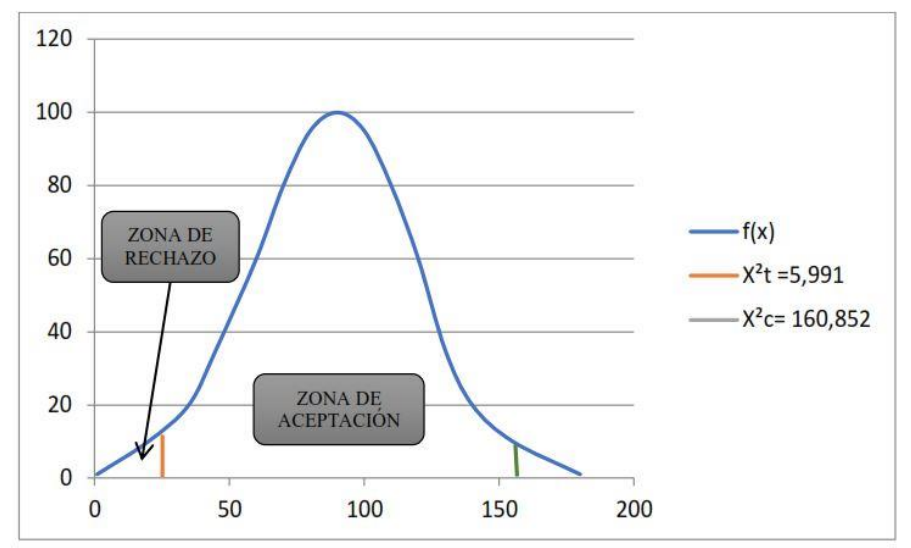

Fuente: Tobar (2021)

De acuerdo con Hernández et al. (2014), La regla para aceptar la hipótesis alternativa dice que el valor del chi cuadrado que se visualiza en la tabla estándar tiene que ser menor al chi cuadrado calculado $\left(\mathrm{X}^{2} \mathrm{t}<\mathrm{X}^{2} \mathrm{c}\right)$. (pág. 212).

Por lo tanto, basado en los cálculos realizados anteriormente se observa los siguientes resultados. $\mathrm{X}^{2} \mathrm{t}=5,991<\mathrm{X}^{2} \mathrm{c}=160,852$

De tal manera que se cumple la regla, por lo que se acepta la hipótesis alternativa (H1), y se rechaza la hipótesis nula (Ho), es así como: La metodología Montessori SI incide en el aprendizaje de la Educación Física online.

Considerando el rango de tiempo comprendido entre el 2015 y el 2020 y según las bases de datos consultadas, se lograron revisar un total de 46 investigaciones, determinándose como estudios potencialmente relevantes un total de 13 investigaciones, de las cuales, el $100 \%$, maneja de forma combinada las variables: estimulación multisensorial temprana y Metodología Montessori.

Si resultó una limitante dentro de la investigación la carencia de estudios específicos, en relación con la funcionabilidad y factibilidad de esta Metodología desde la modalidad online, producto del distanciamiento social; sin embargo se entiende por parte de los autores que aunque los estudios en este sentido aun resultan insuficientes tanto en el contexto internacional como nacional, sí existen evidencias de algunas investigaciones preliminares en esta línea, que acentúan desde la Pedagogía de Montessori, Piaget y otros autores la importancia del juego en el hogar, destacando que en tiempos de confinamiento social el juego constituye una alternativa de invaluable valor para garantizar el desarrollo armónico del niño desde edades tempranas. 
Derivado del análisis se resumen los siguientes aspectos:

- Se sistematiza en la literatura precedente la importancia de la aplicabilidad de la Metodología Montessori en el nivel inicial para el desarrollo de las clases de Educación Física en modalidad online.

- Quedó evidenciado que existen dificultades para el desarrollo de las clases de Educación Física por medio de la modalidad online, entre otros aspectos porque no todos los docentes dominan la metodología y formas adecuadas de enseñanza en esta modalidad y por otra parte porque los estudiantes que integran este nivel manifiestan desmotivación, temor ante la realización de actividades en modalidad online, así como dificultades de conectividad y acceso a la tecnología por lo que requieren acompañamiento familiar.

- Se requiere capacitar a los docentes y padres de familia sobre el uso de la metodología Montessori.

\section{Conclusiones}

Una vez concluida la investigación se determina que:

- La Metodología Montessori es fundamental para el desarrollo de las clases de Educación Física en modalidad online, permite disminuir las brechas existentes entre el nivel de competencias de los docentes y padres de familia para que estén en capacidad de potenciar el desarrollo integral de los estudiantes y al mismo tiempo estimular su participación en las clases.

\section{Referencias Bibliográficas}

Barahona, J. D. (2012). La enseñanza de la Educación Física implementada con TIC. 11.

Bayod, L. (2017) Aula Montessori. www.actividadesparaguarderia.blogspot.com https://www.facebook.com/actividadesparaguarderia

Blández, J. (2005): La asignatura de Educación física de base y una propuesta de adaptación al Espacio Europeo de Educación Superior. Barcelona: INDE.

Estefó, E.C., (2016) Influencia del método Montessori en Educación Física y Salud. Seminario para optar al Título de Profesora de Educación Física, Deportes y Recreación y al Grado de Licenciada en Educación Modalidad: Chile Diciembre, 2016 Tesina 
Fuentes, J. D. (2020). Hacia una educación física pertinente en una región de altitud del Perú en época de pandemia. Revista Peruana de Ciencias de la Actividad Física y del deporte, 997-998.

Gómez G., Rodríguez, C. \& Ramos, M., (2019). Virtual Reality in Physical Education area. Journal of Sport and Health Research. 11(Supl 1):177-186

Hernández, R., Fernández, C., \& Baptista, P. (2014). Metodología de la investigación. http://www.e-libro.com/ayuda

Ministerio de Educación. (2016). Currículo de Educación Física en la EGB y EGU. https://educacion.gob.ec/wp-content/uploads/downloads/2016/08/EFcompleto.pdf

Navacarrada, R., (2010) La Educación Física en el Método Montessori original. Lecturas: Educación Física y Deportes, Revista Digital. Año 14 No 142. Buenos aires marzo 2010 http:// wwwefedeportes.com/

Posso, R.J., Otañez, J.M., Paz, S., Ortiz, A., \& Núñez, L.F.X. (2020). Por una Educación Física virtual en tiempos de COVID. Podium. Revista de Ciencia y Tecnología en la Cultura Física, 15(3), 705-716. Epub 24 de septiembre de 2020. http://scielo.sld.cu/scielo.php?script=sci_arttext\&pid=S1996-

$24522020000300705 \& \operatorname{lng}=$ es\& $\&$ lng=es.

Prieto, J.M., (2020), Recursos Digitales desde la Educacion Fisica. UNIR.NET. https://www.unir.net/educacion/revista/recursos-digitales-educacion-fisica/

Rioja, U. I. (2020), UNIR la universidad en internet. https://www.unir.net/educacion/revista/recursos-digitales-educacion-fisica/

Tobar, B.P., (2021), "La educación física online basada en la metodología Montessori en niños/as de educación inicial II (de 3 - 5 años)” Trabajo de Titulación previo a la obtención del Grado Académico de Magister en Actividad Física Mención Administración y Gestión Deportiva Modalidad de Titulación Proyecto de Desarrollo Humano y Social Integral. Universidad Técnica de Ambato.

Sailema, Á A., Acosta, S. B., Zapata, E. G., \& Estupiñán, M. A. (2021). Estimulación multisensorial temprana desde la metodología Montessori: Reflexiones para su aplicación en condiciones de distanciamiento social. Conciencia Digital, 4(1), 79105. https://doi.org/10.33262/concienciadigital.v4i1.1529

Sánchez, E.D., Ávila, C.M., García, D.G. \& Bravo, W.H. (2020). El proceso de enseñanza aprendizaje de la Educación Física en época de pandemia. Revista: Polo del 
Conocimiento, (Edición núm. 52) Vol. 5, No 11 noviembre 2020, pp. 455-467 ISSN: 2550 - 682X DOI: $10.23857 / p c$. v 5i11.193613-41.

\section{Ciencia
Digital
$\varepsilon$ dituolal}

El artículo que se publica es de exclusiva responsabilidad de los autores y no necesariamente reflejan el pensamiento de la Revista Conciencia Digital.

\section{Ciencia
Digital
Eutuoul}

El artículo queda en propiedad de la revista y, por tanto, su publicación parcial y/o total en otro medio tiene que ser autorizado por el director de la Revista Conciencia Digital.
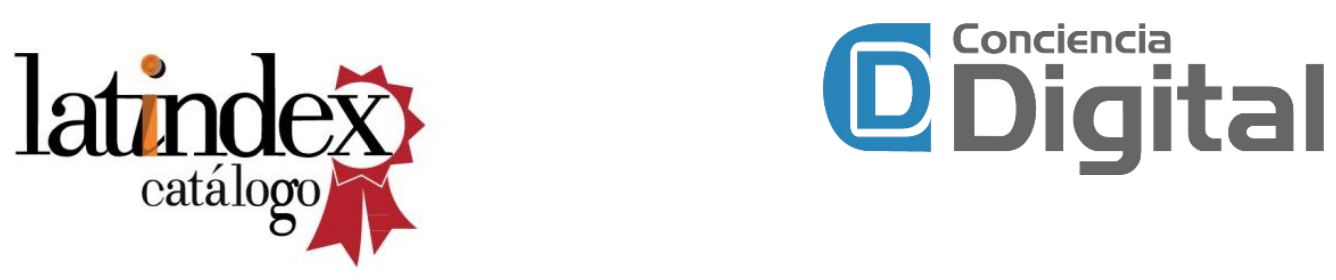

Indexaciones

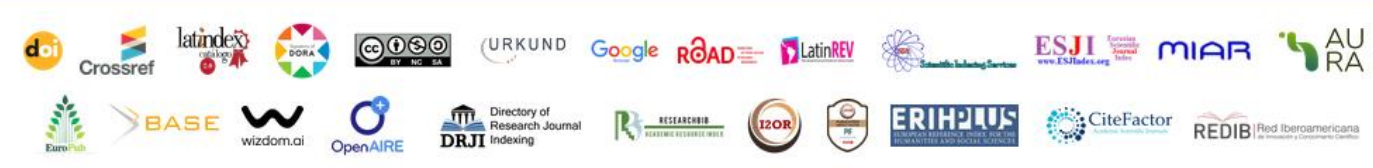

